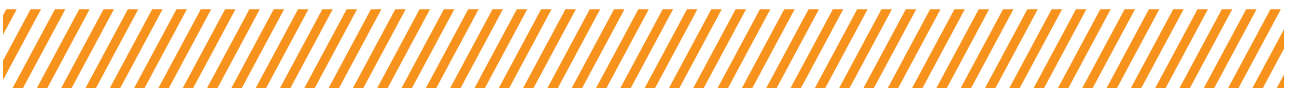

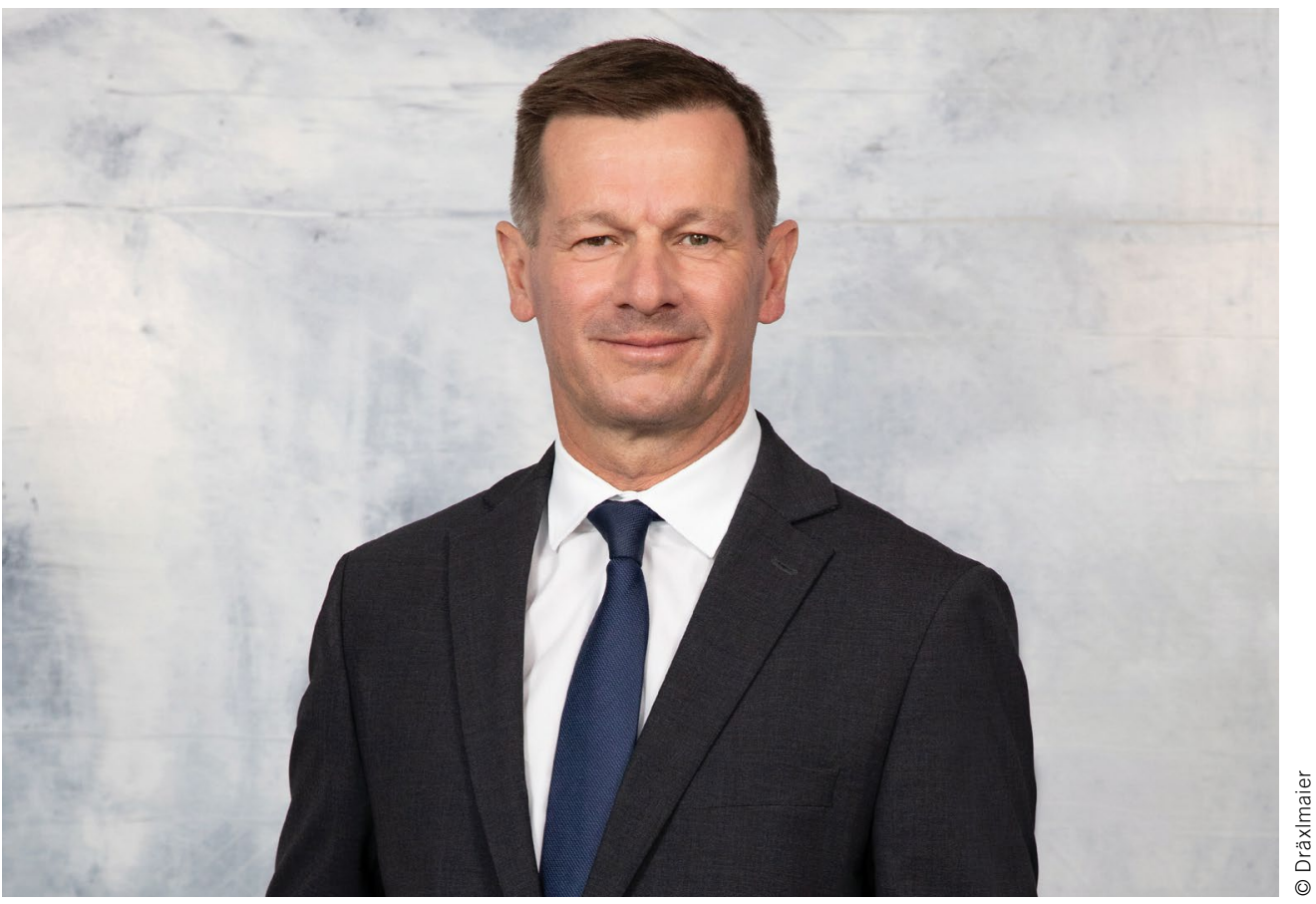

Dr. Martin Gall

CEO und CTO der DräxImaier

Group in Vilsbiburg

\section{Entscheidend ist die Elektronik}

Der Wandel zur Elektromobilität stellt sowohl die Industrie als auch die gesamte Gesellschaft vor große Herausforderungen. Themen wie eine flächendeckende Ladeinfrastruktur und Reichweitenängste bei den Konsumenten stehen dabei immer wieder im Fokus der fachlichen sowie der allgemeinen Diskussion. Sicherlich werden politische Anreize, etwa zur Steigerung der Anzahl von Ladesäulen, ihren Teil zu einer flächendeckenderen Akzeptanz des elektrischen Antriebs beitragen. Aber vor allem die Automobilindustrie selbst kann auf diesem Gebiet in Zukunft mit innovativen technischen Lösungen dafür sorgen, dass die Nutzer zunehmend vom hohen Potenzial der Elektromobilität überzeugt werden.

Der Schlüssel dazu liegt unter anderem in einer gesteigerten Leistungsfähigkeit der eingesetzten Batterieelektronik: Die Batterie selbst kann durch elektrochemische Limitierungen, ihr

Eigengewicht und den angestrebten Kostenrahmen nicht beliebig vergrößert werden. Dagegen ist es möglich, die gespeicherten Energiemengen mittels intelligenter Steuerung und entsprechendem Monitoring noch effizienter und nachhaltiger zu nutzen. Es kommt also darauf an, die zur Verfügung stehende Energie bestmöglich in Vortrieb umzusetzen - und gerade dies wird durch den Einsatz intelligenter und leistungsfähiger Batteriemanagementsysteme möglich, die alle Parameter des Akkus in Echtzeit analysieren und zugleich eine möglichst exakte Restreichweite prognostizieren. Zudem können durch die präzise Kenntnis des aktuellen Zustands aller Batteriekom- ponenten zusätzliche Premiumfunktionen wie etwa eine temporäre Leistungssteigerung des Fahrzeugs ermöglicht werden.

Des Weiteren hat der Einsatz leistungsfähiger Batterieelektronik einen positiven Einfluss auf den ökologischen Fußabdruck des Fahrzeugs: Die Batterie im Fahrzeug wird durch ein ausgeklügeltes Zellmanagement gleichmäßiger belastet, was deren Langlebigkeit zugutekommt. Zudem bildet der durch die Elektronik ermittelte „State of health“ der Komponenten die Grundlage für eine spätere Wiederverwendung des Akkus als stationärer Stromspeicher. Durch die verlängerte Nutzungsdauer wird die Umweltbilanz des Elektroantriebs noch einmal wesentlich verbessert.

Bei einem Blick in die Zukunft ist bereits heute abzusehen, dass das aktuell sehr breite Technologie- und Leistungsfeld der Batteriezellen eine zunehmende Verdichtung erfahren wird, weshalb OEMs bei Großvolumenanwendungen von mehreren Zellherstellern mit gleicher Qualität- und Leistungsfähigkeit beliefert werden können. Die Elektronik spielt hingegen weiter eine große Rolle bei der Schaffung neuer Funktionen, der Effizienzsteigerung des Fahrzeugs sowie der Verbesserung des Komforts für den Fahrer. Eine kontinuierliche Weiterentwicklung der Batteriemanagementsysteme samt der dazugehörigen Elektronikkomponenten und darauf basierender Software birgt somit ein großes Potenzial für die Automotivebranche, auch in Zukunft individuelle Lösungen für die Premiumfahrzeuge von morgen zu entwickeln. 